\title{
SHAPE ANALYSIS OF SYMMETRIC STRUCTURES: QUANTIFYING VARIATION AMONG INDIVIDUALS AND ASYMMETRY
}

\author{
Christian Peter Klingenberg, ${ }^{1}$ Marta Barluenga, ${ }^{2}$ and Axel Meyer ${ }^{3}$ \\ Department of Biology, University of Konstanz, 78457 Konstanz, Germany \\ ${ }^{1}$ E-mail: Christian.Klingenberg@uni-konstanz.de \\ ${ }^{2}$ E-mail: Marta.Barluenga@uni-konstanz.de \\ ${ }^{3}$ E-mail: Axel.Meyer@uni-konstanz.de
}

\begin{abstract}
Morphometric studies often consider parts with internal left-right symmetry, for instance, the vertebrate skull. This type of symmetry is called object symmetry and is distinguished from matching symmetry, in which two separate structures exist as mirror images of each other, one on each body side. We explain a method for partitioning the total shape variation of landmark configurations with object symmetry into components of symmetric variation among individuals and asymmetry. This method is based on the Procrustes superimposition of the original and a reflected copy of each landmark configuration and is compatible with the two-factor ANOVA model customary in studies of fluctuating asymmetry. We show a fully multivariate framework for testing the effects in the two-factor model with MANOVA statistics, which also applies to shapes with matching symmetry. We apply the new methods in a small case study of pharyngeal jaws of the Neotropical cichlid fish Amphilophus citrinellus. The analysis revealed that the symmetric component of variation in the pharyngeal jaws is dominated by the contrast between two alternative trophic morphs in this species and that there is subtle but statistically significant directional asymmetry. Finally, we provide some general recommendations for morphometric studies of symmetric shapes.
\end{abstract}

Key words.-Fluctuating asymmetry, geometric morphometrics, morphological variation, Procrustes superimposition, shape analysis, symmetry.

Received January 29, 2002. Accepted July 5, 2002.

Bilateral symmetry is a pervasive feature of the body plans of most animals, although radial symmetry and other types of symmetry exist as well. Symmetry implies that an organ or part of an organ is repeated in a different orientation or position and, therefore, that the spatial arrangement is strongly patterned and partly redundant. This structure inherent in morphological data from symmetric parts can be used for studies in a variety of biological contexts. An extensive body of work has used fluctuating asymmetry, small differences between corresponding parts on the left and right body sides, to measure the imprecision of developmental systems in response to various stresses and, more contentiously, as an indicator of individual condition (Palmer and Strobeck 1986; Møller and Swaddle 1997). Other studies have focused on the patterns of covariation of asymmetry to investigate morphological integration and modularity (Leamy 1993; Klingenberg and McIntyre 1998; Auffray et al. 1999; Debat et al. 2000; Klingenberg and Zaklan 2000; Klingenberg et al. 2001a; Klingenberg 2002).

The methods of geometric morphometrics combine the powerful and flexible tools of multivariate statistics with explicit consideration of spatial relations of parts and therefore make it possible to investigate morphological variation with direct reference to the anatomical context of the structure under study (e.g., Bookstein 1991, 1996a; Dryden and Mardia 1998). Previous studies have adapted these methods for the study of left-right asymmetry (Auffray et al. 1996; Smith et al. 1997; Klingenberg and McIntyre 1998). The two-factor ANOVA approach for asymmetry studies of conventional length measurements (Leamy 1984; Palmer and Strobeck 1986) has been extended to quantify individual variation and asymmetry in the geometric shape of paired structures (Klingenberg and McIntyre 1998; Auffray et al. 1999).

A procedure to study variation and asymmetry of shapes with internal symmetry was briefly outlined by Bookstein (1996b, pp. 144 ff.) and Auffray et al. (1999, p. 321). This procedure compares the original shapes of specimens to their respective mirror-image copies and thereby makes it possible to partition shape variation into components of symmetric shape and of asymmetry. Mardia et al. (2000) and Kent and Mardia (2001) recently elaborated this method and provided a formal test for directional asymmetry. Here we extend this approach to encompass the full two-factor ANOVA design including fluctuating asymmetry and measurement error (Palmer and Strobeck 1986). We incorporate this method into a framework for analyses of asymmetry for two- and threedimensional data and present improvements that relax the assumptions of the statistical testing procedures published earlier (Klingenberg and McIntyre 1998).

The topic of symmetry is relevant even for morphometric studies that do not focus directly on asymmetry, because nearly perfect bilateral symmetry of morphological structures can lead to statistical problems if it is not taken into account explicitly (Bookstein 1996b, pp. 144 ff.). Therefore, the issue of symmetry is of concern for all morphometric studies of bilaterally symmetric structures. Some authors have included median and paired landmarks in the same analyses without regard for symmetry (e.g., O'Higgins and Jones 1998; Hennessy and Stringer 2002), whereas others have used various techniques for averaging between body sides to obtain halfconfigurations (e.g., Corti et al. 2001; Ponce de León and Zollikofer 2001; Webster et al. 2001). The framework explained here offers a natural way to estimate the axis or plane of symmetry using the complete information in the data and extract components of symmetric shape variation for the many studies in which asymmetry is not the main interest.

We demonstrate the new approach with a dataset from the lower pharyngeal jaw of the Neotropical cichlid fish Am- 

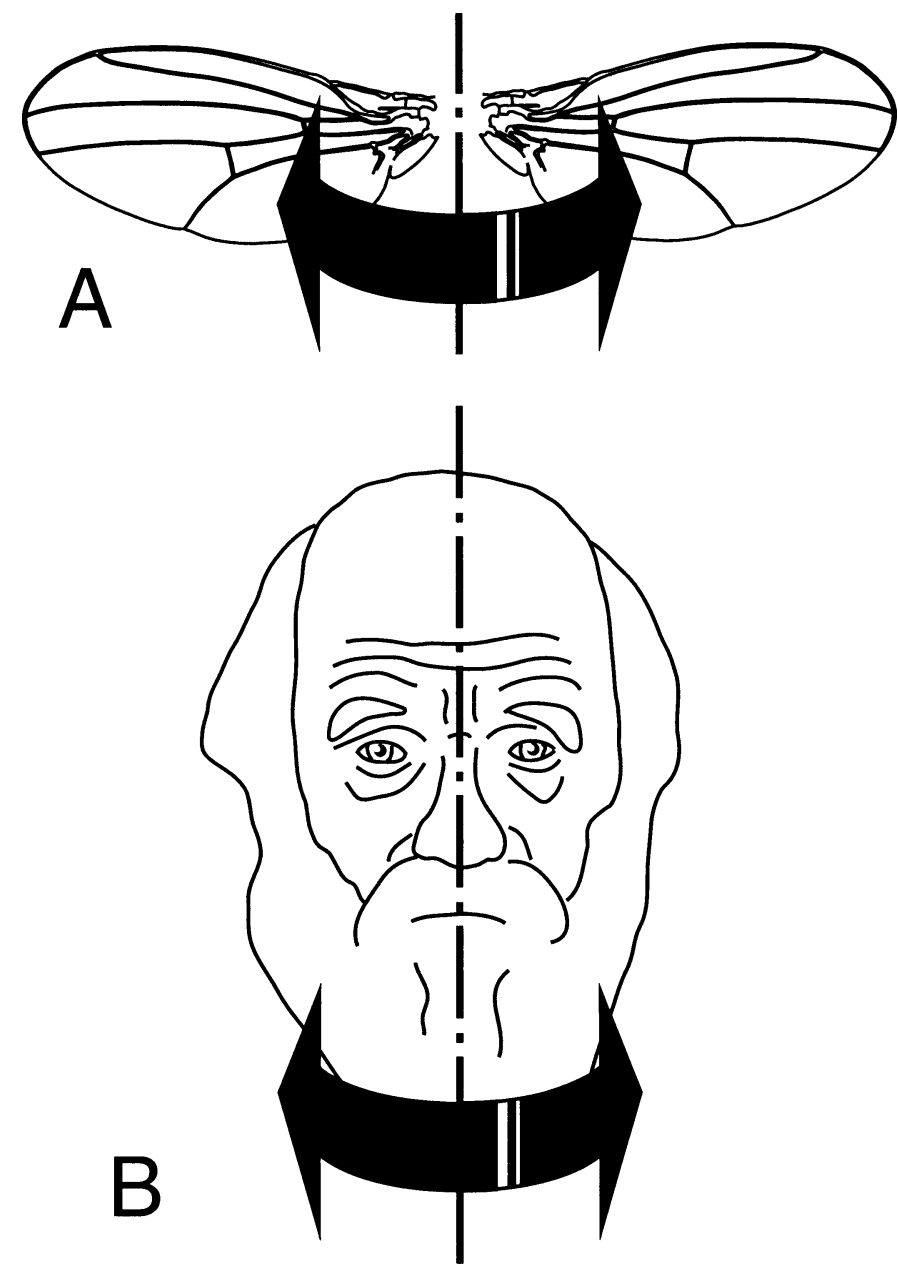

FIG. 1. Examples of the two types of bilateral symmetry (Mardia et al. 2000). (A) Matching symmetry, where a structure is present in two separate copies that are located on the left and right body sides as mirror images of each other. Each copy of the pair is confined entirely to one body side. (B) Object symmetry, where a structure such as the vertebrate head is symmetric in itself because the midsagittal plane (or median plane; the midline in two-dimensional representations) passes through it. Therefore, with the exception of asymmetries, the left and right halves of the structure are mirror images of each other.

philophus citrinellus, which clearly shows internal symmetry and is a structure that is of great interest for cichlid evolution due to its adaptive importance and remarkable phenotypic plasticity (Liem 1973; Meyer 1990a,b; Huysseune et al. 1994; Smits et al. 1996a). Detailed knowledge of the morphological variation in such traits is one of the preconditions for a better understanding of their role in the adaptive evolutionary process.

\section{Types of Symmetry AND ANALYsis OF ASYMmETRY}

\section{Matching Symmetry and Object Symmetry}

Bilateral symmetry can manifest itself in two ways, which Mardia et al. (2000) have distinguished as matching symmetry and object symmetry (Fig. 1). Under matching symmetry, a structure of interest is present in two separate copies as mirror images of one another, one each located on either body side. With object symmetry, the structure is symmetric in itself and therefore has an internal line or plane of symmetry, so that its left and right halves are mirror images of each other.

Each structure with matching symmetry exists as a pair of separate copies, and the plane or axis of symmetry of the animal is located between them, but does not pass through either copy (Fig. 1A). Matching symmetry, as its name suggests, can be assessed by optimally matching the shapes of parts from opposite sides with an appropriate reflection and superimposition. For example, for a comparison of one's left and right hands, this reflection and superimposition corresponds to putting together the palms of both hands. Existing methods for studying asymmetry of shape have focused almost entirely on matching symmetry (e.g., Bookstein 1991, pp. 267 ff.; Auffray et al. 1996; Smith et al. 1997; Klingenberg and McIntyre 1998).

Many parts of biological interest, however, such as the vertebrate skull, have an internal plane of symmetry and thus are bilaterally symmetric in themselves. These structures are instances of object symmetry (Fig. 1B). In addition to the shapes of parts on the left and right sides, the information considered for matching symmetry, studies of object symmetry also analyze variation of structural features in the median plane and the way the two halves of the body are connected. Real organisms hardly ever are perfectly symmetric, however, and small asymmetries also affect those structural features that lie in the midsagittal plane of the idealized body plan. In other words, the surface containing the median landmarks, sutures, and similar midline structures can bulge locally to the left or right to some extent. Therefore studies of variation in symmetric structures need to take into account variation in both the median plane and the paired structures on either side of it.

Even if symmetry or asymmetry are not the primary focus of a study, however, the symmetry in morphological structures is still a concern. It can cause statistical problems, for instance ill-conditioned covariance matrices if all the landmark configurations are very nearly symmetrical (Bookstein $1996 \mathrm{~b}$, pp. $144 \mathrm{ff}$.). If there is exact symmetry, then the positions of the paired landmarks on one body side can be determined from the corresponding landmarks on the other side and those on the median plane. Algebraically, this is a linear dependence among the landmarks, and therefore, the covariance matrix of landmark positions will be singular, which causes difficulties for all statistical procedures that use the inverse or determinant of the dispersion matrix (e.g., canonical variate analysis). Even if symmetry is not perfect, there still can be difficulties because of ill-conditioned covariance matrices. Using complex numbers to analyze landmark coordinates in two dimensions is no remedy to this problem (contrary to the statement of Magwene 2001, p. 646). The problem can be circumvented, however, by taking the symmetry of the forms into account explicitly and adjusting the analysis accordingly.

\section{Procrustes Analysis of Shape}

Most studies in geometric morphometrics characterize shape through the coordinates of a set of landmarks: corre- 
sponding points that can be located unambiguously on each of the specimens under study. A mathematical definition of shape includes all the features of a configuration of landmarks except its size, position, and orientation relative to the original coordinate system. In the context of matching symmetry, reflection is another feature that is not relevant for the study of shape. Procrustes methods eliminate these extraneous features to produce an estimate of mean shape and the variation around the mean (for details, see Rohlf and Slice 1990; Goodall 1991; Bookstein 1996a; Dryden and Mardia 1998; Klingenberg and McIntyre 1998).

Shape analyses of symmetric structures use the generalized least-squares Procrustes superimposition method (Rohlf and Slice 1990; Goodall 1991; Dryden and Mardia 1998). Leastsquares fits are preferable over the resistant-fit methods (Rohlf and Slice 1990) because the algebra underlying the Procrustes fit ties in with the least-squares approach inherent in the ANOVA models used in asymmetry studies. Moreover, for analyses of object symmetry, the repeated median method that is used for resistant fitting (Rohlf and Slice 1990) may not obtain a symmetric consensus from the superimposition of a configuration and its mirror image, and therefore resistant-fit methods may not properly characterize the component of symmetric variation.

\section{Identifying Components of Symmetric Variation and Asymmetry}

Methods for the analysis of shape with bilateral symmetry can separate a component of symmetric variation among individuals, that is, variation in the left-right averages of a trait, from the left-right asymmetries within individuals. Depending on whether the part under study shows matching or object symmetry, there are some differences in the procedure for shape analysis.

\section{Matching symmetry}

For matching symmetry, there are separate landmark configurations for the left and right sides that are mirror images of each other. Therefore, the analysis includes an additional preliminary step: reflecting all configurations from one body side to their mirror images (e.g., by changing the sign of the $x$ coordinate of every landmark for configurations from the left side; Klingenberg and McIntyre 1998). After this, all configurations are superimposed simultaneously with the least-squares Procrustes method.

Because there is a complete landmark configuration on each body side, partitioning of variation into components of symmetric variation among individuals and asymmetry uses averages and contrasts of those configurations. Variation among individuals is analyzed using the averages of the left and right configurations, and asymmetry is measured from the differences between configurations from the left and right sides of each individual. Measurement error is assessed by digitizing each landmark configuration at least twice and computing the differences between replicates of each configuration (Palmer and Strobeck 1986; Palmer 1994; Klingenberg and McIntyre 1998).

Because all of these components of variation are computed from averages or differences of entire landmark configura- tions, they all occupy the same shape space. The dimensionality of this space, called the shape dimension, corresponds to the number of parameters that can be chosen freely to specify a shape or shape difference. For two-dimensional (2D) data, a configuration of $k$ landmarks is defined by $2 k$ coordinates, but these also determine the size, location, and orientation the configuration. Therefore, the number of parameters for these extra sources of variation (eliminated by the Procrustes fit) must be removed: one for size, two for location, and one for orientation. The shape dimension for two-dimensional data is therefore $2 k-4$ (e.g., Goodall 1991). For three-dimensional (3D) data, there are $3 k$ coordinates, and one parameter needs to be removed for scaling, three for location, and three for orientation. The shape dimension for a landmark configuration in $3 \mathrm{D}$ is therefore $3 k$ -7 .

\section{Object symmetry}

For object symmetry, a single landmark configuration provides information about asymmetry and about the left-right average of shape. For each individual, there is a configuration consisting of $k$ pairs of landmarks and $l$ unpaired landmarks on the median plane or midline of the structure, where $k \geq$ $1, l \geq 0$, and $2 k+l \geq 3$ (this notation differs slightly from that of Mardia et al. 2000; Kent and Mardia 2001). For the paired landmarks, which are situated outside of the median plane, the analysis is comparable to matching asymmetry, except that each landmark configuration includes the paired landmarks for both the left and right sides. Therefore, a single configuration contains all the information that analyses of matching symmetry consider separately for the left and right sides, but it also contains additional information on the arrangement of the two halves relative to each other. For a configuration with $k$ pairs of landmarks, the analysis of object symmetry includes all $2 k$ of them simultaneously, whereas a corresponding analysis of matching symmetry deals with only $k$ landmarks at a time, but includes twice the number of configurations. Of course, the unpaired landmarks are unique to analyses of object symmetry.

The analyses for landmark configurations with object symmetry partition the total shape variation into components of symmetric variation and asymmetry by Procrustes superimposition of the original configurations and their mirror images (Fig. 2; Mardia et al. 2000). Before the superimposition, a reflected copy of each configuration is generated (e.g., by reversing the signs of the $x$ coordinate of each landmark). Because the reflection is done before the Procrustes fit, it does not matter which particular plane of reflection is used for each specimen (usually this step is done with the coordinate axes of the original measurements). Then the paired landmarks of the reflected copies are relabeled so that each paired landmark obtains the label of its counterpart (in the example of Fig. 2: $3 \rightarrow 4$ and $4 \rightarrow 3,5 \rightarrow 6$ and $6 \rightarrow 5,7$ $\rightarrow 8$ and $8 \rightarrow 7$ ). The reflection first brings the landmarks to the opposite side, and the relabeling makes the arrangement of landmarks compatible with the original forms again (e.g., in Fig. 2, landmark 3 is to the right of the median axis in the original as well as in the reflected and relabeled configuration). The Procrustes analysis includes the original and 


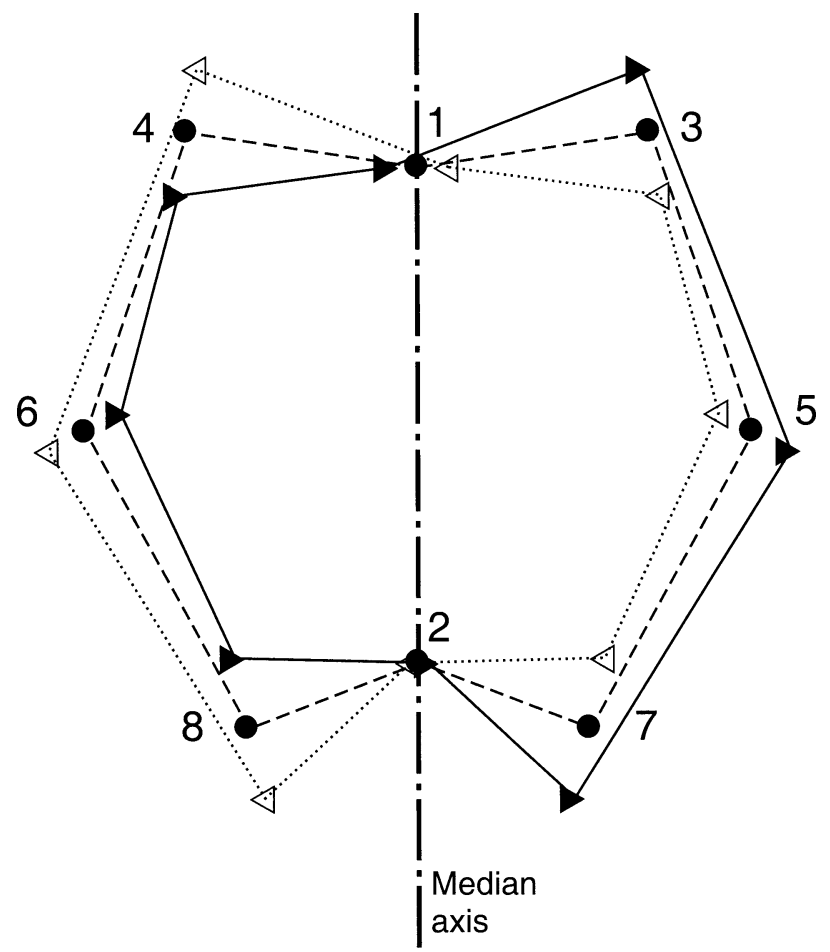

FIG. 2. Analysis of shape variation in structures with object symmetry. The example illustrates the approach with a single arbitrary configuration of eight landmarks. Two are single median landmarks $(1,2)$, whereas the remaining six are in three pairs $(3$ and 4,5 and 6,7 and 8). The diagram shows the original configuration (filled triangles and solid line) and its reflected and relabeled copy (hollow triangles and dotted line) after least-squares Procrustes superimposition. Their Procrustes average (circles and dashed line) is perfectly symmetric. In this average configuration, all the unpaired landmarks and the midpoints between corresponding landmarks on either side fall on a straight line, which is a natural estimate of the median axis (bold dash-dotted line) that allows for asymmetry in all landmarks. The entire coordinate system after the Procrustes fit is rotated so that the median axis of the consensus is vertical.

mirrored configurations of a sample combined, and superimposes all of them simultaneously.

Even for asymmetric shapes, the least-squares Procrustes consensus of a set of landmark configurations and their relabeled mirror images is a perfectly symmetric shape (Fig. 2; Mardia et al. 2000; Kent and Mardia 2001). In this symmetric average, the unpaired landmarks and the midpoints between pairs of landmarks all lie exactly on a straight line (or in a plane for 3D data). Likewise, the average of the original and mirrored configurations of each specimen after the overall Procrustes superimposition is perfectly symmetric. For all these symmetric averages, the unpaired landmarks and the midpoints between pairs of landmarks lie on the same line (or in the same plane) as for the overall consensus. This is a natural estimate of the median axis (or plane) that allows for asymmetry in all landmarks, and provides a frame of reference with a clear anatomical meaning.

The variation among individuals in these averages of original and reflected configurations (corresponding to the leftright averages for matching symmetry) constitutes the symmetric component of shape variation. Symmetric variation includes movements of the unpaired landmarks along the median axis (or within the median plane) and joint mirrorimage shifts of pairs of corresponding landmarks in any direction. For this component of perfect symmetry, the $k$ pairs of landmarks can be fully described by the position of one member of each pair, which is free to vary in any direction, so that there are $2 k$ free parameters for $2 \mathrm{D}$ or $3 k$ free parameters for 3D. The $l$ unpaired landmarks can vary in their positions along the midline or in the median plane and therefore add another $l$ free parameters for $2 \mathrm{D}$ data or $2 l$ for $3 \mathrm{D}$ data. However, the Procrustes fit introduces a number of constraints. The constraint due to size of the whole configuration reduces the number of parameters by one, the constraint for location removes one parameter for $2 \mathrm{D}$ and two for $3 \mathrm{D}$, and an additional constraint for orientation in the median plane removes one more parameter in $3 \mathrm{D}$ (but none in 2D). The shape dimension for variation of symmetric configurations is therefore $2 k+l-2$ for $2 \mathrm{D}$ and $3 k+2 l-4$ for $3 \mathrm{D}$ (see also Mardia et al. 2000; Kent and Mardia 2001).

Asymmetry is quantified through the differences between the original and mirrored configurations, or equivalently, the landmark deviations of the original configuration from the symmetric consensus of the original and mirror image. For the paired landmarks, asymmetry can be in any direction for each of the $k$ pairs, which amounts to $2 k$ free parameters for $2 \mathrm{D}$ and $3 k$ for $3 \mathrm{D}$. Asymmetry of the unpaired landmarks can only be in the direction perpendicular to the midline or median plane and thus adds $l$ free parameters for both 2D and $3 \mathrm{D}$. However, the sum of the asymmetries of all landmarks in the direction perpendicular to the median plane must be zero, eliminating one degree of freedom for both 2D and 3D. Moreover, the rotation step during Procrustes fitting imposes an additional constraint (e.g., it is not possible that all left landmarks shift forward and all right ones backward) and reduces the number of degrees of freedom by one for $2 \mathrm{D}$ and by two for $3 \mathrm{D}$. Notice that there are no constraints for orientation or size of the configurations of the paired landmarks on either side, because differences in size and in orientation of these half-configurations are genuine components of shape asymmetry in structures with object symmetry (e.g., in Fig. 2 , the original configuration's right half is bigger than the left half). Altogether, the shape dimension for asymmetry is therefore $2 k+l-2$ in $2 \mathrm{D}$ or $3 k+l-3$ in $3 \mathrm{D}$.

The shape dimensions of the symmetric and asymmetric components sum up to the total shape dimension for the entire configuration of landmarks, that is, $2(2 k+l)-4$ for $2 \mathrm{D}$ and $3(2 k+l)-7$ for 3D. This is because the components of symmetric variation and asymmetry occupy subspaces of the Procrustes tangent space that are orthogonal complements to each other (for further details on the geometry of this decomposition, see Mardia et al. 2000).

If each configuration has been digitized multiple times, the variation among replicates gives a measure of digitizing error. This error can affect each of the $2 k+l$ landmarks in any direction regardless of the symmetry of the configuration, although the covariance structure may be influenced by the local surroundings of the landmarks. Because the difference between replicates is based on Procrustes superimposition of the whole configuration, the shape dimension for measurement error is $2(2 k+l)-4$ for $2 \mathrm{D}$ and $3(2 k+l)-7$ for $3 \mathrm{D}$. 


\section{Procedures and Tests for Procrustes ANOVA}

The most usual form of this separation is a two-factor ANOVA with individuals and sides as the main effects (Leamy 1984; Palmer and Strobeck 1986; Palmer 1994). This ANOVA approach was originally designed for linear distance measurements, but has been extended for shape data (Klingenberg and McIntyre 1998; Auffray et al. 1999).

The ANOVA design is similar for structures with matching and object symmetry. The ANOVA partitions the deviations of the configurations from the overall consensus (mean) configuration into components due to individuals, sides (or reflections, for structures with object symmetry), individuals $\times$ sides interaction, and measurement error. Variation among individuals, corrected for the effects of any asymmetry, is quantified by the main effect of individuals. Directional asymmetry, the average difference between the two body sides, corresponds to the main effect of sides (or reflections). Fluctuating asymmetry, the variability of left-right differences among individuals, is the individuals $\times$ sides interaction (or individuals $\times$ reflections interaction). Measurement error is computed from the variation among replicate measurements.

\section{Isotropic model}

Goodall (1991, p. 314 ff.) first presented a framework for Procrustes ANOVA of shape data, which has been used in analyses of asymmetry (Klingenberg and McIntyre 1998). This model makes the assumption that there is an equal amount of variation around each landmark, this variation is isotropic (the scatter of residuals is circular in 2D or spherical in $3 \mathrm{D}$, i.e., there are no preferred directions of variation at any landmark), and variation is independent among landmarks. Therefore, analyses can concentrate exclusively on the amount and ignore the direction of variation.

To assess the magnitudes of effects due to different sources of shape variation, it is possible simply to add up the sums of squares for the different effects across the coordinates of all landmarks and to divide by the respective degrees of freedom (derived from the corresponding shape dimensions; see Table 1) to compute Procrustes mean squares (e.g., Klingenberg and McIntyre 1998; Auffray et al. 1999). These mean squares are in units of squared Procrustes distance, and therefore are directly compatible with the algebra that forms the core of statistical shape analysis (Bookstein 1996a; Dryden and Mardia 1998). This measure of the magnitude of different ANOVA effects is particularly useful, for instance, for assessing the relative importance of digitizing error or the contribution of fluctuating asymmetry to overall variation.

A simple procedure for Procrustes ANOVA under the isotropic model consists of the following steps. First, the landmark configurations, prepared as appropriate for matching or object symmetry, are superimposed by the least-squares Procrustes method. Then, a standard statistics package is used to perform a separate two-factor ANOVA of each coordinate, according to the model for univariate studies of asymmetry (Palmer and Strobeck 1986; Palmer 1994). Individually, the ANOVA statistics of single coordinates should be interpreted with the utmost caution, if at all, because they are not independent of one another due to the overall Procrustes fit.

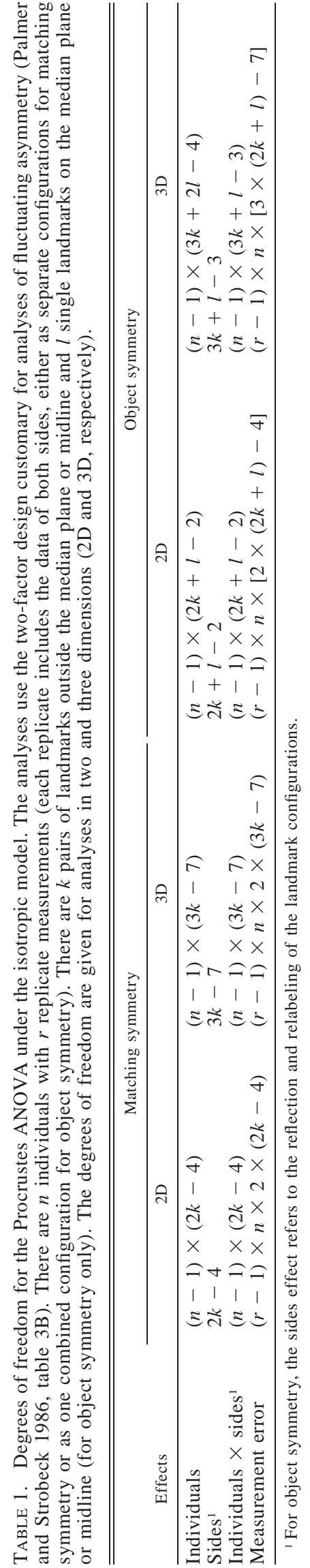


Nevertheless, the sums of squares for each effect can be added over the coordinates of all landmarks, and the Procrustes mean squares are obtained by dividing by the appropriate degrees of freedom (Table 1). Statistical significance can be assessed with parametric $F$-tests (Goodall 1991) or permutation tests (Klingenberg and McIntyre 1998).

The model of equal and isotropic variation is a fairly restrictive assumption that is often violated in biological datasets, which often show clearly patterned variation (e.g., Klingenberg and McIntyre 1998; Badyaev and Foresman 2000; Debat et al. 2000; Klingenberg and Zaklan 2000; Klingenberg and Leamy 2001; Klingenberg et al. 2001a). Therefore, although the Procrustes mean squares provide a useful and appropriate measure of the magnitude of effects, further interpretations of ANOVA results should be made with caution. In particular, significance levels obtained by parametric $F$-tests may not be reliable. Permutation tests (Good 1994; Edgington 1995) provide an alternative that can be used for Procrustes ANOVA even when distributions are nonnormal or sample size is small (Klingenberg and McIntyre 1998), but as far as these procedures also sum variation across landmarks and coordinates, they also rely on the same assumption.

\section{Nonisotropic model}

To avoid making the assumption of equal, independent, and isotropic variation at all landmarks, Mardia et al. (2000) proposed a fully multivariate test of directional asymmetry for the case of object symmetry (see also Kent and Mardia 2001). This test is a comparison of the original versus the reflected and relabeled landmark configuration based on the $T^{2}$ statistic, using the mean squares of the individuals $\times$ reflection effect as the error covariance matrix (for matching symmetry, the test would be a comparison of the left and right sides).

It is possible to extend this multivariate approach to the full two-factor MANOVA corresponding to the univariate design (Palmer and Strobeck 1986; Palmer 1994). This means that matrices of sums of squares and cross-products (SSCP) are computed for each effect, and compared with the standard statistics for MANOVA (e.g., Seber 1984). There are some specific problems, however, concerning the dimensionality of shape variation.

For matching symmetry, the covariance matrices for all effects of the model span a space of $2 k-4$ or $3 k-7$ dimensions (in $2 \mathrm{D}$ or $3 \mathrm{D}$, respectively), and therefore less than the number of coordinate axes. Because the standard MANOVA tests involve determinants or inverses (e.g., Seber 1984), which require full-rank SSCP matrices (at least for the error effect), it is important that they are carried out in the Procrustes tangent space, using a coordinate system with the appropriate dimensionality (Dryden and Mardia 1998; Rohlf 1999). An alternative is to modify the standard test statistics by using the generalized inverse so that they can tolerate singular matrices. For instance, the Lawley-Hotelling trace $T_{g}^{2}$ (Seber 1984, pp. $38 \mathrm{ff}$.), modified in this way, is the sum of the diagonal elements of the matrix $\mathbf{B W}^{-}$multiplied by the degrees of freedom for the error effect (where $\mathbf{B}$ is the SSCP matrix for the effect being tested, $\mathbf{W}^{-}$is the Moore-
Penrose generalized inverse of the SSCP matrix for the error effect). For MANOVA statistics like Wilks' lambda (Seber 1984, p. 435), for which determinants of SSCP matrices must be computed, the product of the nonzero eigenvalues of the respective matrix is used instead of the determinant. Overall, however, the MANOVA for matching symmetry is a fairly straightforward generalization of the univariate model: all the effects are computed from averages or contrasts in the same shape space (e.g., left-right differences).

The situation is somewhat more complex for object symmetry, because the components of symmetric variation and asymmetry occupy different subspaces of the shape (tangent) space for the entire landmark configurations. Particularly, the test for the main effect of individuals, consisting of symmetric variation, cannot use the individual $\times$ reflection interaction as the error effect because that interaction is part of the asymmetry component and therefore in a different subspace. An alternative would be to assume an arbitrary error model, for instance, with isotropic variation of the same magnitude as for fluctuating asymmetry at each landmark. However, this is somewhat at odds with the purpose of MANOVA, whose aim is to avoid precisely that kind of assumption. Another alternative would be to test the main effect of individuals over the symmetric component of measurement error, but a test assessing individual variation against measurement imprecision rather than against the developmental variability of the structure would address a quite different question. Nevertheless, whether variation among individuals is statistical significant is rarely the primary question in analyses of asymmetry, and it may therefore often be possible to omit this test altogether.

The MANOVA test for directional asymmetry is more straightforward; it is based on the main effect of reflection and can use the individual $\times$ reflection interaction as the error effect. Both these effects use the asymmetry component of variation and are therefore in the same shape subspace. Modified MANOVA statistics based on the generalized inverse should be used because these SSCP matrices are not of full rank. This MANOVA test of directional asymmetry using the Lawley-Hotelling trace statistic $T_{g}^{2}$ is the same as the $T^{2}$ test proposed by Mardia et al. (2000) and by Kent and Mardia (2001) because the $T_{g}^{2}$ reduces to Hotelling's $T^{2}$ statistic if there is only one degree of freedom for the effect being tested (Seber 1984, p. 44).

The test for fluctuating asymmetry, which compares the individual $\times$ reflection interaction to measurement error, is also possible with the modified MANOVA statistics. However, measurement error is estimated from the total variation among replicates of the entire landmark configurations and therefore includes symmetric variation as well as an asymmetry component of measurement error. The appropriate measure of error against which to gauge fluctuating asymmetry is the precision with which left-right differences in the configuration can be measured. Therefore, the error effect to test fluctuating asymmetry is the asymmetry component of the measurement error, which is derived from the differences between replicate measurements in the differences between the original and reflected configurations. 


\section{Comparison of Covariance Structure}

For each of the effects included in the Procrustes ANOVA, there is a matrix of mean squares and cross products or of variance and covariance components that can be used for analyses of the patterns of covariance. Comparisons of the covariance structure of individual variation (left-right means) and of fluctuating asymmetry are relevant to a range of questions relating to the developmental origins of shape variation. Agreement or differences in the patterns of shape variation can be used as evidence of whether the same developmental processes are involved at both levels of variation (Klingenberg and McIntyre 1998; Badyaev and Foresman 2000; Debat et al. 2000). Moreover, covariance of fluctuating asymmetry can be used to infer domains within which direct developmental interactions prevail and therefore to delimit developmental modules (Klingenberg and Zaklan 2000; Klingenberg et al. 2001a; Klingenberg 2002).

All the published comparisons of shape covariance structure between individual variation and fluctuating asymmetry have focused on parts with matching symmetry (or have treated the data as for matching symmetry). For this kind of data, the analysis is straightforward because the analyses of asymmetry and left-right means are carried out in the same shape space. The comparison is between the covariance matrix of left-right averages and the one for left-right differences (Klingenberg and McIntyre 1998; Debat et al. 2000; Klingenberg and Zaklan 2000).

For structures with object symmetry, however, the components of symmetric variation and asymmetry occupy complementary subspaces in the tangent space defined by the whole configurations, and all the features of symmetry variation are therefore orthogonal to the features of asymmetry and vice versa. Thus, direct comparisons of the covariance structure are not informative, and an alternative procedure is required. A possibility is to concentrate on half-configurations and to analyze the corresponding parts of the covariance matrices for fluctuating asymmetry and individual variation (i.e., after a Procrustes fit of the whole original configurations and relabeled reflections). Because variation of the unpaired landmarks is partitioned into complementary components of symmetric variation within the median plane and of asymmetry perpendicular to the median plane, these landmarks are not informative in this context (but they provide information on the axis or plane of symmetry in the overall Procrustes fit). Therefore, the comparison of covariance matrices must be restricted to the paired landmarks. The symmetry and asymmetry components for the paired landmarks of one body side are each specified by $2 k$ coordinates for $2 \mathrm{D}$ data and by $3 k$ coordinates for $3 \mathrm{D}$ data. The information in these data is almost the same as would be included in an analysis of the paired landmarks within the framework for matching symmetry, but it contains additional information about the arrangement of the two half-configurations.

An overall comparison of these partial covariance matrices can be made by matrix correlation and statistical significance can be assessed with a Mantel test (Mantel 1967; Cheverud et al. 1989; Sokal and Rohlf 1995, pp. 813 ff.) adapted specifically for geometric morphometrics (Klingenberg and McIntyre 1998, p. 1367). The modifications reflect that the landmarks, not single coordinates, are the central items in geometric morphometrics. A difference from the standard procedure is that the diagonals of the matrices are included in the computation of matrix correlation because the variation of each landmark coordinate is informative as well (alternatively, if the diagonal is to be excluded, entire diagonal blocks should be removed including the covariances between $x, y$, and possibly $z$ coordinates of each landmark). Moreover, the Mantel test is based on random permutations of the landmarks, not of single variables, and therefore leaves the coordinates of each landmark associated with each other (for additional details see Klingenberg and McIntyre 1998). If the Mantel test rejects the null hypothesis of total independence of the matrices, further comparisons of the covariance structures can be made, for instance, by comparing principal components (PCs) (e.g., Klingenberg and McIntyre 1998; Klingenberg and Zaklan 2000).

\section{Case Study: Materials and Methods}

\section{Specimens and Measurements}

The lower pharyngeal jaw of cichlid fishes is fused from the left and right fifth ceratobranchials, which are separate structures in generalized percoid fishes (Liem 1973; Galis and Drucker 1996). The midsagittal surface where the two halves are sutured together is therefore a plane of symmetry for the pharyngeal jaw, which is a typical example of object symmetry. We used a sample of 40 pharyngeal jaws of the Neotropical cichlid A. citrinellus, for which substantial morphological variation associated with trophic polymorphism has been demonstrated (Meyer 1990a,b). This is a small subset of a larger dataset that will be analyzed elsewhere.

Images of the dorsal side of the pharyngeal jaws were produced with a flatbed scanner at a resolution of $600 \mathrm{dpi}$. Nine landmarks on the outline of each jaw (Fig. 3) were digitized with the aid of a macro written in Object-Image (software freely available from http://simon.bio.uva.nl/ object-image.html). One of these landmarks is on the midline (landmark 1), whereas the others are arranged in four pairs on the left and right sides. Each pharyngeal jaw was scanned twice, so that the error associated with the imaging and digitizing procedures could be quantified.

\section{Analyses}

To examine the relative amounts of symmetric variation and asymmetry, we used Procrustes ANOVA (Klingenberg and McIntyre 1998), as adapted for studies of object symmetry (see above). In this analysis we added sums of squares across landmarks and coordinates and thus assumed equal and isotropic variation at each landmark. To establish the statistical significance of the various effects, we conducted both parametric tests based on $F$-statistics (Goodall 1991) and permutation tests (Good 1994; Edgington 1995) following the procedures for asymmetry studies (Klingenberg and McIntyre 1998). For the main effect of individuals, the null hypothesis was simulated by randomly permuting the landmark configurations among individuals, but keeping the original and reflected configurations separate. For the main effect of reflections, the original and reflected configurations were 


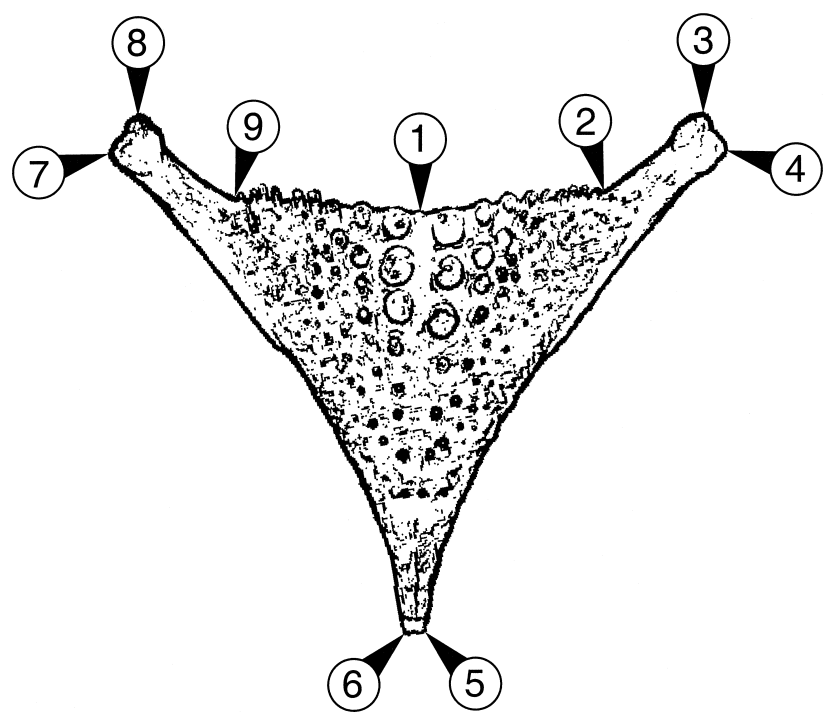

FIG. 3. Landmarks used in the study of variation in lower pharyngeal jaws of the cichlid Amphilophus citrinellus, an example of object symmetry. Landmark 1 is on the midline, whereas the remaining landmarks are arranged in symmetric pairs. The lower pharyngeal jaw is composed of the pair of fifth ceratobranchials joined at the midline (Liem 1973; Huysseune et al. 1994). It has a central tooth-bearing plate surrounded by an anterior extension (bottom) and two postero-lateral horns that serve as muscular attachment sites.

randomly swapped for each individual. Finally, the null hypothesis of no interaction term was simulated by first removing both main effects by subtraction of means and then permuting configurations among individuals and reflections (Good 1994). For each effect of the model, the test consisted of 10,000 rounds of permutation.

The statistic for testing MANOVA effects under the nonisotropic model was the Lawley-Hotelling trace $T_{g}^{2}$ (Seber 1984, pp. 38 ff.), modified by the use of the Moore-Penrose generalized inverse. For the parametric tests, significance levels were computed by an approximation via the $F$-distribution (Seber 1984, p. 39). In addition, we also conducted permutation tests as outlined above, but with the $T_{g}^{2}$ statistic.

For each of the MANOVA effects, patterns of shape variation were visualized with PCs of the respective matrix of mean squares and cross-products. To compare the covariance structure between the components of symmetric variation among individuals and of fluctuating asymmetry, we restricted the analysis to the paired landmarks of one side (landmarks 2-5). A Mantel test of matrix correlation was used for the comparison of the modified covariance matrices, with per-
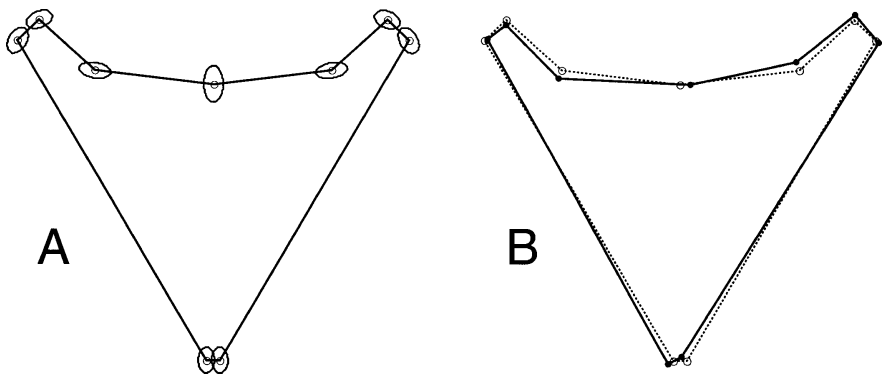

FIG. 4. Average shape and directional asymmetry. (A) The Procrustes consensus for all original and reflected configurations, with 95\% equal density ellipses to show the scatter of landmark positions around the overall average. This configuration is perfectly symmetric. (B) Directional asymmetry, shown as the difference between the averages of all original and reflected configurations and amplified five-fold for better visibility (solid line and solid circles). For comparison, the diagram shows the symmetric overall Procrustes consensus (dotted line and open circles).

mutations over the landmarks. Because there were only four pairs of landmarks, it was feasible to base the test on a full enumeration of all possible permutations, and the $P$-values are therefore exact.

\section{RESULTS}

Procrustes superimposition of all original and reflected configurations produced a symmetric consensus configuration (Fig. 4A). Partitioning the variation around this consensus by Procrustes ANOVA (Table 2) indicated that variation in symmetric shape among individuals accounts for the largest portion of the total variation, but that there is also a considerable amount of fluctuating asymmetry (individual $\times$ reflection interaction). Variation of left-right means among individuals and fluctuating asymmetry are highly significant statistically. Directional asymmetry is subtle, so that it needed to be amplified for graphical display (Fig. 4B), and its statistical significance is ambiguous because the $P$-value from the parametric $F$-test is somewhat below and that from the permutation test slightly above the conventional 5\% significance threshold. On the whole, however, the results of the parametric and permutation tests agree.

The assumption of isotropic variation at each landmark is questionable because the scatters of landmark positions around the overall consensus are not circular (Fig. 4A). Consequently, MANOVA tests will be more appropriate than the isotropic model. MANOVA tests, carried out with both parametric and permutation methods (Table 3), indicate that directional asymmetry is also highly significant. Because it

TABLE 2. Procrustes ANOVA for the pharyngeal jaws, a structure that shows object symmetry. Sums of squares (SS) and mean squares (MS) are in dimensionless units of Procrustes distance (for the derivation of degrees of freedom, see Table 1). This analysis adds the sums of squares over landmarks and coordinates and thereby assumes that all landmarks have the same amount of isotropic variation.

\begin{tabular}{|c|c|c|c|c|c|c|}
\hline Source & $\mathrm{df}$ & SS & MS & $F$ & $\begin{array}{c}P \\
\text { (parametric) }\end{array}$ & $\begin{array}{c}P \\
\text { (permutation) }\end{array}$ \\
\hline Individual & 273 & 0.16742 & 0.0006133 & 4.68 & 0 & $<0.0001$ \\
\hline Reflection & 7 & 0.00204 & 0.0002909 & 2.22 & 0.033 & 0.057 \\
\hline Measurement error & 560 & 0.00897 & 0.0000160 & & & \\
\hline
\end{tabular}


TABLE 3. MANOVA tests for directional asymmetry and fluctuating asymmetry of the pharyngeal jaws. The error effect used to test the main effect of reflection is the individual $\times$ reflection interaction, whereas the interaction effect was tested against the asymmetry component of the among-image variation (replicate measurements). The test statistic used is the Lawley-Hotelling trace, $T_{g}^{2}$ (Seber 1984). The test for the main effect of reflection is identical to the test of directional asymmetry proposed by Mardia et al. (2000). Notice that, due to object symmetry, there is no test for the main effect of individuals corresponding to the univariate test (Palmer and Strobeck 1986), because the individual $\times$ reflection interaction occupies a different subspace of the shape space for the whole landmark configurations.

\begin{tabular}{lclc}
\hline \hline & & \multicolumn{2}{c}{$P$} \\
\multicolumn{1}{c}{ Source } & $T_{g}^{2}$ & $\begin{array}{c}\text { (para- } \\
\text { metric) }\end{array}$ & $\begin{array}{c}P \\
\text { (permutation) }\end{array}$ \\
\hline Reflection & 43.14 & 0.007 & 0.0002 \\
Individual $\times$ reflection & 12521 & 0 & $<0.0001$ \\
\hline
\end{tabular}

avoids the assumption of equal and isotropic variation at each landmark and thus takes into account the structure of shape variation, the MANOVA test has higher statistical power. It is pertinent that the largest landmark shifts due to directional asymmetry (e.g., at landmarks 2 and 9) are in directions for which the respective landmarks show relatively little variation (cf. Figs. 4A and B). The test for the individual $\times$ re-
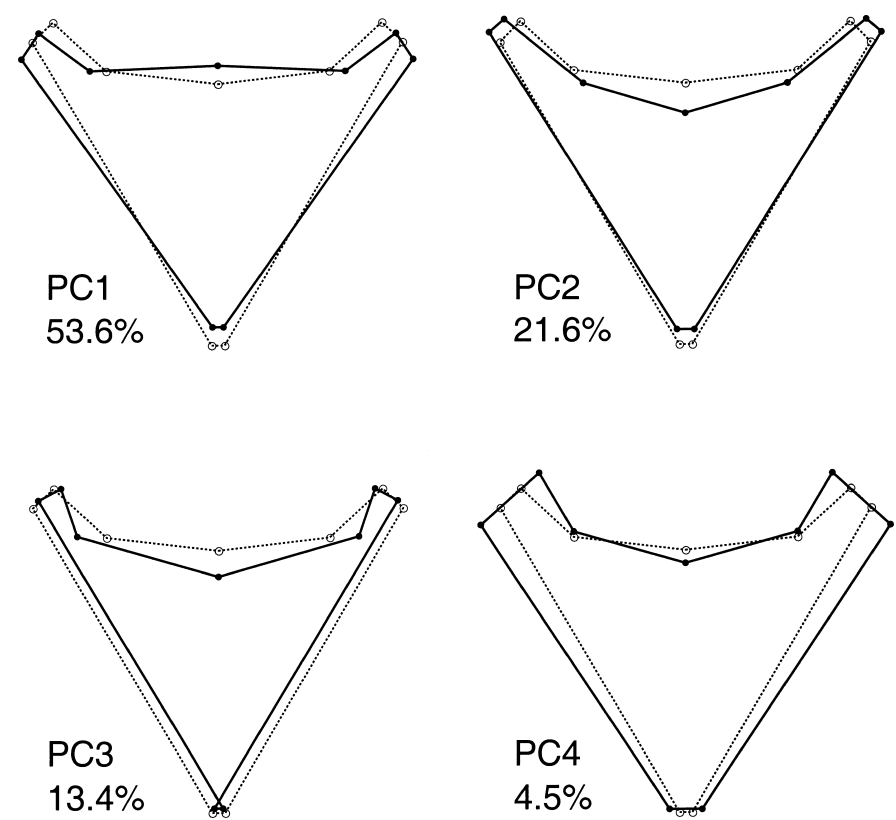

FIG. 5. Patterns of the symmetric part of shape variation. The diagrams show the first four principal components (PCs) of the matrix of mean squares and cross products for variation among individuals, that is, variation in the averages of the original and reflected landmark configurations. Therefore, all the variation displayed here is constrained to symmetry. For each PC, the diagram shows the change from the overall consensus configuration (dotted lines, open circles) to a configuration with an arbitrary PC score of +0.1 units of Procrustes distance (solid lines, solid circles). These shape changes are exaggerated in comparison to those in the data (e.g., for PC 3 landmarks 5 and 6 even cross over through the median plane). The sign for PCs is arbitrary, and the reverse shape change would therefore be equivalent. The percentages indicate the share of the total symmetric component of shape variation for which each PC accounts.
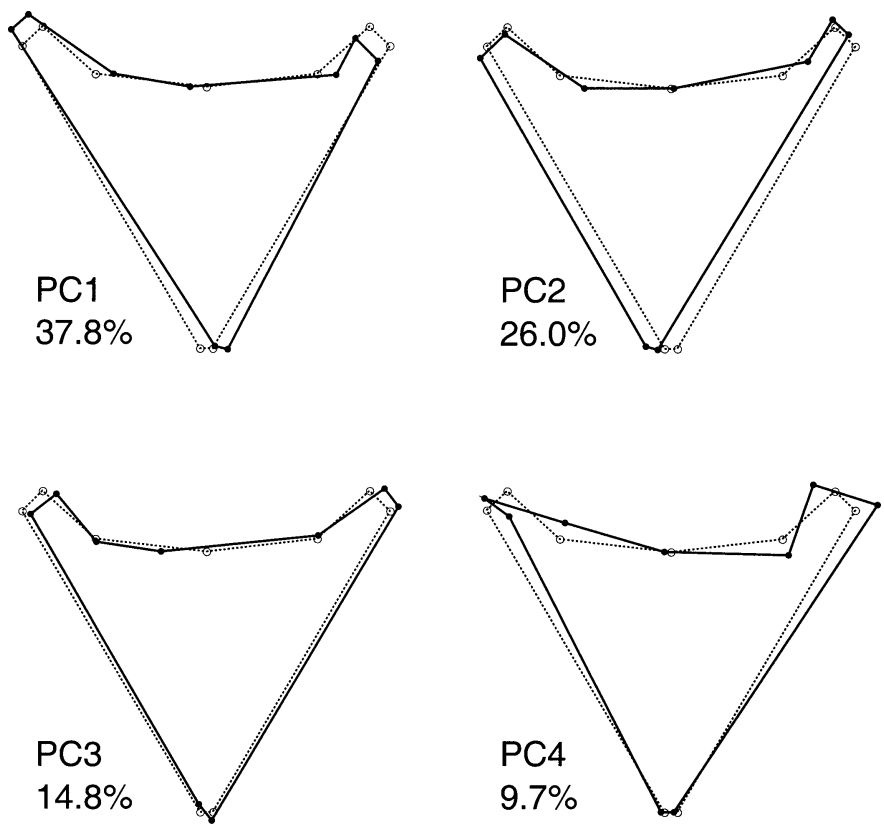

FIG. 6. Patterns of fluctuating asymmetry. The figure shows the first four principal components (PCs) of the covariance matrix for the individual $\times$ side interaction effect of the MANOVA model. In other words, this is the variation of individual asymmetries around the average (directional) asymmetry shown in Figure 4B. The diagrams show the shape change from the symmetric consensus configuration (dotted lines, open circles) to a configuration with an arbitrary score of +0.1 units of Procrustes distance for the respective PC (solid lines, solid circles). The signs for PCs are arbitrary, and the mirror images of the PC patterns shown here would therefore be equivalent. The percentages indicate the share of the total variation associated with the individual $\times$ side interaction for which each PC accounts.

flection interaction yields a highly significant result with both methods, because the amount of fluctuating asymmetry greatly exceeds that of measurement error.

Principal component analysis was used to identify and display the patterns of individual variation and fluctuating asymmetry. The analysis of symmetric variation shows that the PC1 alone takes up more than half of the variation (Fig. 5). Compared to the consensus, a pharyngeal jaw shape with a positive PC1 score has a wider angle at the anterior tip, lateral horns that are shorter and more robust, and a more convex (or less concave) posterior contour near landmark 1 (Fig. 5). This pattern of variation agrees well with a change from papilliform to a more molariform jaw shape, and we therefore interpret the PC1 as a contrast between the two morphs of pharyngeal jaws. The PC2 is primarily associated with an extension of the lateral horns at the expense of the toothbearing area and anterior tip, and the PC 3 and PC4 primarily feature different aspects of the robustness of the lateral horns and anterior tip (Fig. 5).

The variation due to fluctuating asymmetry is spread more evenly over multiple dimensions than is symmetric variation, as indicated by the percentages of the total variation taken up by the PCs of the matrix of mean squares and crossproducts for the individual $\times$ reflection interaction (Fig. 6). The dominant features of variation concern asymmetry in the placement of landmark 1 relative to the neighboring land- 
marks 2 and 9 on the posterior edge of the tooth-bearing plate, the angle between this edge and the lateral horns, and the length and thickness of the horns.

Comparison of the PCs for symmetric variation among individuals (Fig. 5) and for fluctuating asymmetry (Fig. 6) suggests little agreement of the patterns of variation. As outlined above, comparison of patterns of symmetric variation and of asymmetry must be restricted to the four paired landmarks. The matrix correlation, including diagonal elements, was 0.46 but not statistically significant $(P=0.83$; computed through enumeration of all possible permutations of landmarks). This moderately high value of the matrix correlation results largely from the diagonal elements being greater than the off-diagonal elements, that is, the variation at each landmark exceeds the covariation among landmarks. Accordingly, the matrix correlation drops to $-0.12(P=0.79)$ if the diagonal blocks of the matrices (the variances of landmark coordinates and the covariances between the $x$ and $y$ coordinates of each landmark) are omitted. Because there is no significant relationship between these covariance matrices, we do not present any more detailed comparisons.

\section{Discussion}

In this paper, we have used the distinction of Mardia et al. (2000) between matching symmetry, where separate parts from the left and right body sides are compared, and object symmetry, where the structure itself has an internal axis or plane of symmetry. The paper has introduced new methodology for studying shape variation and asymmetry in structures with object symmetry, extending the approach offered by Mardia et al. (2000) and earlier methods for studies of matching symmetry (e.g., Auffray et al. 1996; Smith et al. 1997; Klingenberg and McIntyre 1998; Auffray et al. 1999; Klingenberg and Zaklan 2000). We have demonstrated the new methodology in a small case study of shape variation in the pharyngeal jaws from cichlid fishes.

\section{Components of Shape Variation in Pharyngeal Jaws}

The lower pharyngeal jaw of cichlid fishes, consisting of the pair of fifth ceratobranchials sutured together in the median plane (e.g., Liem 1973), is a typical structure with object symmetry. The analysis has demonstrated the partitioning of the shape variation of the whole configuration of landmarks into complementary components of symmetric variation among individuals and of asymmetry.

The pharyngeal jaws of $A$. citrinellus show subtle, but statistically significant directional asymmetry (Fig. 4B). The increased statistical power offered by the MANOVA test, allowing a sharper detection of differences in directions with smaller variability, has revealed this result for the present dataset. This finding parallels those from studies of other structures like mouse teeth, skulls, and mandibles (Auffray et al. 1996; Debat et al. 2000; Klingenberg et al. 2001b; Leamy et al. 2001) or insect wings (Smith et al. 1997; Klingenberg et al. 1998, 2001a). On the one hand, this result underscores the apparent ubiquity of directional asymmetry in animals (Klingenberg et al. 1998) and is in agreement with the rapidly increasing knowledge about consistent left-right asymmetries that have been reported from the development of all major model animals (Tamura et al. 1999; Capdevila et al. 2000; Ligoxygakis et al. 2001). On the other hand, the ability to find such subtle effects with high statistical significance emphasizes the need to take every possible precaution against systematic errors in the data-acquisition process (e.g., parallax error from using stereo microscopes or other optical distortion during the imaging steps). Any such systematic error might well appear as a statistically significant but spurious finding of directional asymmetry. Careful checks and calibration of all optical equipment can guard against this sort of artifact.

Over half of the symmetric component of shape variation is associated with the PC1 (Fig. 5), which can be interpreted as the contrast between papilliform and molariform morphs of pharyngeal jaws associated with the trophic polymorphism in this species (Meyer 1990a) and other cichlids (Huysseune et al. 1994; Smits et al. 1996a,b). Fish of the molariform morph are more effective at crushing snails and accordingly eat such hard prey items more frequently than do fish of the papilliform morph, which are more efficient at processing soft prey (Meyer 1989). As indicated by the names, the teeth on the pharyngeal jaws are bigger and molar-shaped in the molariform morph, but smaller and more pointed in the papilliform morph (Meyer 1990b). But in addition, according to their use in crushing hard prey items, molariform pharyngeal jaws are more robust than papilliform ones, with relatively shorter and stouter muscle attachment processes and an enlarged tooth-bearing plate; these are the shape changes that appear in the PC1 (Fig. 5). There is considerable phenotypic plasticity in pharyngeal jaw morphology (Meyer 1990a; Huysseune et al. 1994). Accordingly, it is not entirely clear to what extent the shape variation represented by the PC1 has a direct genetic basis and to what extent it is a consequence of the relative numbers of different prey items eaten by the fish.

A prominent role of phenotypic plasticity may also explain the discrepancy in the patterns of covariation between the symmetric shape variation among individuals and fluctuating asymmetry. Differences in diet that influence the expression of morphological variation would simultaneously affect both body sides, and therefore contribute to the symmetric component of variation. Its effect thus would be qualitatively different from those developmental processes that operate concurrently in the growing structures on either body sides, but without direct linkage, and therefore may contribute to both symmetric variation and asymmetry. Behavioral variation and habitual asymmetries in the handling of prey may contribute to further variation and asymmetry of pharyngeal jaw shapes, for instance in the length or robustness of muscle attachment processes and in the relative size of the toothbearing plate, as they are represented by the PC2-PC4 of symmetric variation (Fig. 5) and the PCs of fluctuating asymmetry (Fig. 6). In this way, phenotypic plasticity in response to diet composition would cause a qualitative difference between the processes affecting the symmetric and asymmetric components of variation (see also Debat et al. 2000).

These results should be interpreted with caution, however, and need to be reevaluated with a larger dataset. The small sample size limits the statistical power, and the small number of landmarks limits the spatial information available for the 
analyses. Particularly for the comparison of the symmetric component of variation and fluctuating asymmetry, the comparison is based on only four pairs of landmarks (with additional constraints imposed). The purpose of this paper was to introduce and demonstrate new methods, but to address the biological questions raised here, these methods will have to be applied in a more extensive study.

\section{Recommendations for the Study of Symmetric Shapes}

The geometry of many morphological structures has an internal symmetry, that is, they show object symmetry. Single landmark configurations from such parts provide information about both symmetric variation and asymmetry, and morphometric analyses of such configurations should take this data structure into account. On the one hand, this avoids statistical problems in the analyses (e.g., Bookstein 1996b, pp. 143 ff.) and unambiguously separates variation among specimens from intra-individual asymmetries. On the other hand, the alignment in the median plane provides morphometric studies with a clear frame of reference that relates directly to the anatomy of the organism.

Studies that focus on the variation among individuals, where asymmetry is not of particular interest, will focus on the component of symmetric shape variation. For each landmark configuration, a reflected and relabeled duplicate is added to the dataset, a simultaneous Procrustes fit is done for all the configurations, and subsequent analyses are based on the averaged Procrustes coordinates of each specimen and its mirror image. This provides a multivariate space of the appropriate dimensionality to study shape variation. The dimensionality is $2 k+l-2$ for $2 \mathrm{D}$ data or $3 k+2 l-4$ for $3 \mathrm{D}$ data, even though there will be more coordinates for the configurations. This limited dimensionality should be taken into account particularly in statistical tests. In addition, multivariate analyses that involve the inverse of the covariance matrix, for example, canonical variate analysis, discriminant analysis or MANOVA, should be modified by the use of the generalized inverse (e.g., Dryden and Mardia 1998, p. 152). Even though asymmetry may not be the focus of interest, the asymmetric component of variation can still be useful as a measure of developmental variability and measurement error that can be used as a standard for comparison with the symmetric variation among individuals.

The implications are even more immediate for studies of asymmetry. Object symmetry is different from matching symmetry because information about asymmetry is contained in the single configuration of landmarks. There is no separate analysis for the asymmetry of size, as for matching symmetry, because differences in the size of the left and right halves of a configuration are part of the overall shape variation of configurations with object symmetry. Nevertheless, the two-factor ANOVA customary for asymmetry studies can be applied with some modest modifications. As we showed, however, the comparison of covariance structure between fluctuating asymmetry and the symmetric variation is more difficult for configurations with object symmetry than for those with matching symmetry.

\section{ACKNOWLEDGMENTS}

We thank H. Fischer and A. Merkel for help with the preparation of pharyngeal jaws, as well as F. L. Bookstein and K. V. Mardia for discussion and for providing preprints of papers. We thank J.-C. Auffray, V. B. DeLeon, R. J. Hennessy, L. J. Leamy, P. C. Wainwright, and two anonymous reviewers for their helpful comments and questions on earlier drafts of the manuscript.

\section{Literature Cited}

Auffray, J.-C., P. Alibert, S. Renaud, A. Orth, and F. Bonhomme. 1996. Fluctuating asymmetry in Mus musculus subspecific hybridization: traditional and Procrustes comparative approach. Pp. 275-283 in L. F. Marcus, M. Corti, A. Loy, G. J. P. Naylor, and D. E. Slice, eds. Advances in morphometrics. Plenum Press, New York.

Auffray, J.-C., V. Debat, and P. Alibert. 1999. Shape asymmetry and developmental stability. Pp. 309-324 in M. A. J. Chaplain, G. D. Singh, and J. C. McLachlan, eds. On growth and form: spatio-temporal pattern formation in biology. Wiley, Chichester, U.K.

Badyaev, A. V., and K. R. Foresman. 2000. Extreme environmental change and evolution: stress-induced morphological variation is strongly concordant with patterns of evolutionary divergence in shrew mandibles. Proc. R. Soc. Lond. B Biol. Sci. 267:371-377.

Bookstein, F. L. 1991. Morphometric tools for landmark data: geometry and biology. Cambridge Univ. Press, Cambridge, U.K.

- 1996a. Biometrics, biomathematics and the morphometric synthesis. Bull. Math. Biol. 58:313-365.

- 1996b. Combining the tools of geometric morphometrics. Pp. 131-151 in L. F. Marcus, M. Corti, A. Loy, G. J. P. Naylor, and D. E. Slice, eds. Advances in morphometrics. Plenum Press, New York

Capdevila, J., K. J. Vogan, C. J. Tabin, and J. C. Izpisúa Belmonte. 2000. Mechanisms of left-right determination in vertebrates. Cell 101:9-21.

Cheverud, J. M., G. P. Wagner, and M. M. Dow. 1989. Methods for the comparative analysis of variation patterns. Syst. Zool. 38:201-213.

Corti, M., M. Aguilera, and E. Capanna. 2001. Size and shape changes in the skull accompanying speciation of South American spiny rats (Rodentia: Proechimys spp.). J. Zool. 253:537-547.

Debat, V., P. Alibert, P. David, E. Paradis, and J.-C. Auffray. 2000. Independence between developmental stability and canalization in the skull of the house mouse. Proc. R. Soc. Lond. B Biol. Sci. 267:423-430.

Dryden, I. L., and K. V. Mardia. 1998. Statistical analysis of shape. Wiley, Chichester, U.K.

Edgington, E. S. 1995. Randomization tests. Marcel Dekker, New York.

Galis, F., and E. G. Drucker. 1996. Pharyngeal biting mechanics in centrarchid and cichlid fishes: insights into a key evolutionary innovation. J. Evol. Biol. 9:641-670.

Good, P. 1994. Permutation tests: a practical guide to resampling methods for testing hypotheses. Springer-Verlag, New York.

Goodall, C. R. 1991. Procrustes methods in the statistical analysis of shape. J. R. Stat. Soc. B 53:285-339.

Hennessy, R. J., and C. B. Stringer. 2002. Geometric morphometric study of the regional variation of modern human craniofacial form. Am. J. Phys. Anthropol. 117:37-48.

Huysseune, A., J.-Y. Sire, and F. J. Meunier. 1994. Comparative study of lower pharyngeal jaw structure in two phenotypes of Astatoreochromis alluaudi (Teleostei: Cichlidae). J. Morphol. 221:25-43.

Kent, J. T., and K. V. Mardia. 2001. Shape, Procrustes tangent projections and bilateral symmetry. Biometrika 88:469-485.

Klingenberg, C. P. 2002. Developmental instability as a research tool: using patterns of fluctuating asymmetry to infer the developmental origins of morphological integration. Pp. 427-442 
in M. Polak, ed. Developmental instability: causes and consequences. Oxford Univ. Press, New York.

Klingenberg, C. P., and L. J. Leamy. 2001. Quantitative genetics of geometric shape in the mouse mandible. Evolution 55: 2342-2352.

Klingenberg, C. P., and G. S. McIntyre. 1998. Geometric morphometrics of developmental instability: analyzing patterns of fluctuating asymmetry with Procrustes methods. Evolution 52: $1363-1375$.

Klingenberg, C. P., and S. D. Zaklan. 2000. Morphological integration between developmental compartments in the Drosophila wing. Evolution 54:1273-1285.

Klingenberg, C. P., G. S. McIntyre, and S. D. Zaklan. 1998. Leftright asymmetry of fly wings and the evolution of body axes. Proc. R. Soc. Lond. B Biol. Sci. 265:1255-1259.

Klingenberg, C. P., A. V. Badyaev, S. M. Sowry, and N. J. Beckwith. 2001a. Inferring developmental modularity from morphological integration: analysis of individual variation and asymmetry in bumblebee wings. Am. Nat. 157:11-23.

Klingenberg, C. P., L. J. Leamy, E. J. Routman, and J. M. Cheverud. 2001b. Genetic architecture of mandible shape in mice: effects of quantitative trait loci analyzed by geometric morphometrics. Genetics 157:785-802.

Leamy, L. 1984. Morphometric studies in inbred and hybrid house mice. V. Directional and fluctuating asymmetry. Am. Nat. 123: 579-593.

- 1993. Morphological integration of fluctuating asymmetry in the mouse mandible. Genetica 89:139-153.

Leamy, L. J., S. Meagher, S. Taylor, L. Carroll, and W. K. Potts. 2001. Size and fluctuating asymmetry of morphometric characters in mice: their associations with inbreeding and the $t$-haplotype. Evolution 55:2333-2341.

Liem, K. F. 1973. Evolutionary strategies and morphological innovations: cichlid pharyngeal jaws. Syst. Zool. 22:425-441.

Ligoxygakis, P., M. Strigini, and M. Averof. 2001. Specification of left-right asymmetry in the embryonic gut of Drosophila. Development 128:1171-1174.

Magwene, P. M. 2001. Comparing ontogenetic trajectories using growth data. Syst. Biol. 50:640-656.

Mantel, N. 1967. The detection of disease clustering and a generalized regression approach. Cancer Res. 27:209-220.

Mardia, K. V., F. L. Bookstein, and I. J. Moreton. 2000. Statistical assessment of bilateral symmetry of shapes. Biometrika 87: 285-300.

Meyer, A. 1989. Cost of morphological specialization: feeding performance of the two morphs in the trophically polymorphic cichlid fish, Cichlasoma citrinellum. Oecologia 80:431-436.

1990a. Ecological and evolutionary consequences of the trophic polymorphism in Cichlasoma citrinellum (Pisces: Cichlidae). Biol. J. Linn. Soc. 39:279-299.

1990b. Morphometrics and allometry in the trophically polymorphic cichlid fish, Cichlasoma citrinellum: alternative adaptations and ontogenetic changes in shape. J. Zool. 221: 237-260.

Møller, A. P., and J. P. Swaddle. 1997. Asymmetry, developmental stability, and evolution. Oxford Univ. Press, Oxford, U.K.

O'Higgins, P., and N. Jones. 1998. Facial growth in Cercocebus torquatus: an application of three-dimensional geometric morphometric techniques to the study of morphological variation. J. Anat. 193:251-272.

Palmer, A. R. 1994. Fluctuating asymmetry analyses: a primer. Pp. 335-364 in T. A. Markow, ed. Developmental instability: its origins and implications. Kluwer, Dordrecht, The Netherlands.

Palmer, A. R., and C. Strobeck. 1986. Fluctuating asymmetry: measurement, analysis, patterns. Annu. Rev. Ecol. Syst. 17:391-421.

Ponce de León, M. S., and C. P. E. Zollikofer. 2001. Neanderthal cranial ontogeny and its implications for late homonid diversity. Nature 412:534-538.

Rohlf, F. J. 1999. Shape statistics: Procrustes superimpositions and tangent spaces. J. Classif. 16:197-223.

Rohlf, F. J., and D. Slice. 1990. Extensions of the Procrustes method for the optimal superimposition of landmarks. Syst. Zool. 39: $40-59$.

Seber, G. A. F. 1984. Multivariate observations. John Wiley and Sons, New York.

Smith, D. R., B. J. Crespi, and F. L. Bookstein. 1997. Fluctuating asymmetry in the honey bee, Apis mellifera: effects of ploidy and hybridization. J. Evol. Biol. 10:551-574.

Smits, J. D., F. Witte, and D. E. Povel. 1996a. Differences between inter- and intraspecific architectoniuc adaptations to pharyngeal mollusc crushing in cichlid fishes. Biol. J. Linn. Soc. 59: 367-387.

Smits, J. D., F. Witte, and F. G. van Veen. 1996b. Functional changes in the anatomy of the pharyngeal jaw apparatus of Astatoreochromis alluaudi (Pisces, Cichlidae), and their effects on adjacent structures. Biol. J. Linn. Soc. 59:389-409.

Sokal, R. R., and F. J. Rohlf. 1995. Biometry: the principles and practice of statistics in biological research. W. H. Freeman, New York.

Tamura, K., S. Yonei-Tamura, and J. C. Izpisúa Belmonte. 1999. Molecular basis of left-right asymmetry. Dev. Growth Differ. 41:645-656.

Webster, M., H. D. Sheets, and N. C. Hughes. 2001. Allometric patterning in trilobite ontogeny: testing for heterochrony in $\mathrm{Ne}$ phrolenellus. Pp. 105-144 in M. L. Zelditch, ed. Beyond heterochrony: the evolution of development. Wiley-Liss, New York.

Corresponding Editor: P. Wainwright 